Claremont Colleges

Scholarship@ Claremont

All HMC Faculty Publications and Research

HMC Faculty Scholarship

4-1-2004

\title{
Double Excitations within Time-Dependent Density Functional Theory Linear Response
}

Neepa T. Maitra

Rutgers University - New Brunswick/Piscataway

Fan Zhang

Rutgers University - New Brunswick/Piscataway

Robert J. Cave

Harvey Mudd College

Kieron Burke

Rutgers University - New Brunswick/Piscataway

\section{Recommended Citation}

Double excitations within time-dependent density functional theory linear response. Neepa T. Maitra, Fan Zhang, Robert J. Cave, and Kieron Burke, J. Chem. Phys. 120, 5932 (2004), DOI:10.1063/1.1651060

This Article is brought to you for free and open access by the HMC Faculty Scholarship at Scholarship @ Claremont. It has been accepted for inclusion in All HMC Faculty Publications and Research by an authorized administrator of Scholarship @ Claremont. For more information, please contact scholarship@cuc.claremont.edu. 


\title{
Double excitations within time-dependent density functional theory linear response
}

\author{
Neepa T. Maitra ${ }^{\text {a) }}$ \\ Department of Physics and Astronomy, City University of New York and Hunter College,
} New York, New York 10021

Fan Zhang

Department of Physics and Astronomy, Rutgers University, Piscataway, New Jersey 08854-8019

Robert J. Cave

Department of Chemistry, Harvey Mudd College, Claremont, California 91711

Kieron Burke

Department of Chemistry and Chemical Biology, Rutgers University, Piscataway, New Jersey 08854

(Received 24 November 2003; accepted 8 January 2004)

\begin{abstract}
Within the adiabatic approximation, time-dependent density functional theory yields only single excitations. Near states of double excitation character, the exact exchange-correlation kernel has a strong dependence on frequency. We derive the exact frequency-dependent kernel when a double excitation mixes with a single excitation, well separated from the other excitations, in the limit that the electron-electron interaction is weak. Building on this, we construct a nonempirical approximation for the general case, and illustrate our results on a simple model. (C) 2004 American Institute of Physics. [DOI: 10.1063/1.1651060]
\end{abstract}

\section{INTRODUCTION}

Ground-state density functional theory (DFT) is an efficient and popular calculational method in solid-state physics and quantum chemistry. ${ }^{1}$ The one-to-one mapping between densities and potentials gives rise to a Kohn-Sham (KS) system of noninteracting electrons, whose equations can be solved much faster than the fully interacting Schrödinger equation, and which yields in principle all the properties of the true interacting system. ${ }^{2,3}$ In practice, approximations must be made for the unknown exchange-correlation (xc) energy, and recent years have seen the development of increasingly accurate functionals, ${ }^{4-6}$ applied to increasingly complex systems. ${ }^{7}$ Time-dependent DFT (TDDFT) is an extension of the ground-state theory to time-dependent potentials. ${ }^{8}$ Today this is most widely used in the linear response regime, where excitations and oscillator strengths of atoms, molecules, and solids are calculated. The excitations of the ground-state KS system are not those of the true system, but in principle, all excitations of the system may be obtained exactly from them using the xc kernel through an integral equation, ${ }^{9}$ or in a matrix formulation. ${ }^{10}$ The xc kernel describes the change in the time-dependent $\mathrm{xc}$ potential when a perturbation is applied to the system, $f_{\mathrm{XC}}\left[n_{0}\right]\left(\mathbf{r}, \mathbf{r}^{\prime}, t-t^{\prime}\right)=\delta v_{\mathrm{XC}}(\mathbf{r} t) /\left.\delta n\left(\mathbf{r} t^{\prime}\right)\right|_{n_{0}} ; \quad$ its Fourier transform to the frequency domain is what is needed for these calculations. Alternatively, one may obtain the excita-

${ }^{a)}$ Electronic mail: nmaitra@hunter.cuny.edu tion energies from the Fourier transform of a real-time calculation where the system is subjected to a weak perturbation. ${ }^{11-13}$

In any of these schemes an adiabatic approximation is usually used, in which the kernel is local in time (or, in the frequency domain, frequency-independent); most often it is simply the functional derivative of the ground-state xc potential used in the calculation of the bare KS transitions. The matrix formulation of TDDFT with an adiabatic functional has been programmed in standard quantum chemical codes, leading to hundreds of calculations of excitations (see, e.g., Ref. 14 for many references). In many cases, the transition frequencies are remarkably accurate. However the errors are generally not well understood (although, see Ref. 15 for some recent progress). The accuracy of the ground-state functional used, especially its asymptotic behavior, has been explored in, for example, Refs. 16 and 17, but much less is known about the validity of the adiabatic approximation for the xc kernel, ${ }^{18-21}$ this understanding is needed in order for TDDFT to be used for calculations of excited states with as much confidence as DFT is used for ground-state properties.

This paper concerns the TDDFT treatment of double excitations, an issue of great practical importance in quantum chemistry, and yet one that has been puzzled over and not well understood. ${ }^{22-25}$ A natural approach is to study higherorder response, ${ }^{26}$ but this is rather cumbersome. The need to understand the role and importance of double excitations is not merely of academic interest. The low-lying electronic states of many conjugated molecules exhibit significant double excitation character in wave function treatments. ${ }^{23,24,27-30} \mathrm{~A}$ classic example is the family of poly- 
enes. The lowest-lying singlet state of all polyenes is not a simple one-electron excitation from the highest occupied to the lowest unoccupied orbital of the ground state, but has a large zeroth-order contribution from a doubly excited determinant. The two-photon absorption characteristics of these systems support an interpretation of these states as largely "doubly excited." 31 As discussed in Sec. II, exact linear response TDDFT in principle produces transitions to excitations of any number, but the integral (or matrix) equations yield simple corrections to the KS response, which contains poles at only single excitations. How are the "extra" poles of the true system generated in the formalism? We show that the exact xc kernel has strong frequency dependence in the vicinity of double excitations that enables TDDFT to generate more poles and include transitions to states with double excitation character. These cannot be captured when an adiabatic approximation is used.

In Sec. III, we derive the exact frequency-dependent xc kernel within a dressed single-pole framework, for the following case: when one double-excitation mixes with a nearby single excitation, well separated from all the other excitations in the system, in the limit that the electronelectron interaction is weak. We build on this result to construct a nonempirical approximation that goes beyond the weak-interaction limit: this, and its generalization to cases when more than one single excitation is nearby, ${ }^{32}$ gives a practical scheme for including double excitations in TDDFT linear response. We discuss when an adiabatic TDDFT (ATDDFT) calculation provides a reasonable approximation for states with a double excitation component. Although we focus on double excitations, since these are of most practical interest, the same ideas can be applied to excitations of any number. Our results are demonstrated on a simple model system in Sec. IV: two fermions in a one-dimensional harmonic well interacting via a delta-function repulsion.

\section{THEORY}

Given a system of $N$ interacting electrons, the groundstate KS potential is defined as the one-body potential in which $N$ noninteracting electrons live so as to produce the exact ground-state density $n_{0}(\mathbf{r})$ of the interacting system. Excitations of this KS system are not however those of the interacting system. Instead, the linear response theory of TDDFT provides a route to obtaining excitation energies and oscillator strengths of the interacting system, from knowledge of only the ground-state KS potential.

Applying a small perturbing potential to a ground state, and measuring the density response defines the susceptibility, or density-density response function, $\chi\left[n_{0}\right]\left(\mathbf{r}, \mathbf{r}^{\prime}, t-t^{\prime}\right)$ $=\delta v_{\text {ext }}(\mathbf{r} t) /\left.\delta n\left(\mathbf{r}^{\prime} t^{\prime}\right)\right|_{n_{0}}$. The susceptibility of the true interacting system is related to that of its noninteracting Kohn-Sham counterpart, $\quad \chi_{s}\left[n_{0}\right]\left(\mathbf{r}, \mathbf{r}^{\prime}, t-t^{\prime}\right)=\delta v_{s}(\mathbf{r} t) /$ $\left.\delta n\left(\mathbf{r}^{\prime} t^{\prime}\right)\right|_{n_{0}}$, through an integral equation, written in the frequency domain as ${ }^{9}$

$$
\stackrel{\leftrightarrow}{\chi}^{-1}(\omega)=\stackrel{\leftrightarrow}{\chi}_{s}^{-1}(\omega)-\stackrel{\leftrightarrow}{f}_{\mathrm{HXC}}(\omega) .
$$

Here the Hartree-exchange-correlation kernel $\stackrel{\leftrightarrow}{f}_{\mathrm{HXC}}(\omega)$ is the sum of the Hartree kernel and the xc kernel defined ear- lier, $f_{\mathrm{HXC}}\left[n_{0}\right]\left(\mathbf{r}, \mathbf{r}^{\prime}, \omega\right)=f_{\mathrm{H}}\left(\mathbf{r}, \mathbf{r}^{\prime}\right)+f_{\mathrm{XC}}\left[n_{0}\right]\left(\mathbf{r}, \mathbf{r}^{\prime}, \omega\right)$; the Hartree kernel is the density-functional-derivative of the Hartree potential, $f_{\mathrm{H}}\left(\mathbf{r}, \mathbf{r}^{\prime}\right)=1 /\left|\mathbf{r}-\mathbf{r}^{\prime}\right|$. Transition frequencies of the true system lie at the poles of $\chi\left(\mathbf{r}, \mathbf{r}^{\prime}, \omega\right)$, and oscillator strengths may be obtained from the residues. The poles of $\chi_{s}\left(\mathbf{r}, \mathbf{r}^{\prime}, \omega\right)$ are at the KS single excitations; these are shifted to the true excitations through the action of the Hartree-exchange-correlation kernel. So, Eq. (1) enables us to obtain the interacting excitation energies and oscillator strengths from the KS susceptibility and the Hartreeexchange-correlation kernel. In principle, the exact spectrum of the interacting system is obtained; in practice, approximations must be made for (a) the xc contribution to the ground-state KS potential, and (b) the xc kernel $f_{\mathrm{XC}}(\omega)$.

In the Lehman representation,

$$
\chi\left(\mathbf{r}, \mathbf{r}^{\prime} ; \omega\right)=\sum_{I}\left\{\frac{F_{I}(\mathbf{r}) F_{I}^{*}\left(\mathbf{r}^{\prime}\right)}{\omega-\omega_{I}+i 0^{+}}-\frac{F_{I}^{*}(\mathbf{r}) F_{I}\left(\mathbf{r}^{\prime}\right)}{\omega+\omega_{I}+i 0^{+}}\right\},
$$

where, $F_{I}(\mathbf{r})=\langle 0|\hat{n}(\mathbf{r})| I\rangle$ with $\hat{n}(\mathbf{r})$ being the one-body density operator, $I$ labels the excited states of the interacting system, and $\omega_{I}$ is their transition frequency. This expression also holds for the KS susceptibility where the excited states are excited Slater determinants and the transition frequencies are orbital energy differences.

Due to the one-body nature of the density operator $\hat{n}(\mathbf{r})$ in the numerator, $\chi_{s}$ has poles only at single KS excitations. For the interacting system, where exact eigenstates may be mixtures of single, double, and higher-multiple excitations, the susceptibility contains poles at states dominated by any number of excitations. So, in the bound spectrum, $\chi_{s}$ has fewer poles than $\chi$. The failure of ATDDFT to generate more poles and produce double excitations is most easily seen in Casida's matrix formulation. ${ }^{10}$ Let $q=(i, a)$ be an index representing a single excitation: a transition from an occupied $\mathrm{KS}$ orbital $\phi_{i}$ to an unoccupied one $\phi_{a}$, and let $\omega_{q}$ be the difference in the KS orbital energies, $\omega_{q}=\epsilon_{a}-\epsilon_{i}$. Then, the squares of the true transition frequencies $\Omega_{I}=\omega_{I}^{2}$ are the eigenvalues of the matrix

$$
\widetilde{\Omega}(\omega)_{q q^{\prime}}=\delta_{q q^{\prime}} \omega_{q}^{2}+4 \sqrt{\omega_{q} \omega_{q^{\prime}}}\left[q\left|f_{\mathrm{HXC}}(\omega)\right| q^{\prime}\right],
$$

where

$$
\begin{aligned}
{\left[q\left|f_{\mathrm{HXC}}(\omega)\right| q^{\prime}\right]=} & \int d \mathbf{r} d \mathbf{r}^{\prime} \boldsymbol{\phi}_{i}^{*}(\mathbf{r}) \phi_{a}(\mathbf{r}) \\
& \times f_{\mathrm{HXC}}\left(\mathbf{r}, \mathbf{r}^{\prime}, \omega\right) \phi_{i^{\prime}}\left(\mathbf{r}^{\prime}\right) \phi_{a^{\prime}}^{*}\left(\mathbf{r}^{\prime}\right) .
\end{aligned}
$$

Oscillator strengths of the true system are related to the eigenvectors. ${ }^{10}$ Because the matrix in Eq. (3) spans only the $\mathrm{KS}$ single excitations, if $f_{\mathrm{HXC}}$ is frequency independent, the number of eigenvalues of the matrix is equal only to the number of single excitations: multiple excitation information cannot be gained in the adiabatic approximation. ${ }^{22}$

Before resolving this problem we note that a useful approximation for the true transition frequencies is obtained by expanding the linear response equation, Eq. (1), around each KS transition frequency., 93 This "single pole approximation" (SPA) can alternatively be derived from neglecting the 
off-diagonal terms in Casida's matrix and assuming the correction due to $f_{\mathrm{HXC}}$ is small compared with the bare KohnSham value. One finds

$$
\omega=\omega_{q}+2\left[q\left|f_{\mathrm{HXC}}\left(\omega_{q}\right)\right| q\right] .
$$

The SPA corrects the single excitations of the KS system toward the true ones, and, in many cases, especially those of atoms and molecules where the transitions are "well separated," 15 the SPA value lies very close to the full TDDFT value.

Consider the situation of a KS single excitation, $\phi_{i}$ $\rightarrow \phi_{a}$, of frequency $\omega_{q}=\epsilon_{a}-\epsilon_{i}$, close to a double excitation, and well separated from all other levels. Let the true excitations, $\Psi_{a}, \Psi_{b}$, near this frequency be mixtures of the single and double, such that as the electron-electron interaction strength $\lambda \rightarrow 0$,

$$
\begin{aligned}
& \Psi_{a}=m \Phi_{D}+\sqrt{1-m^{2}} \Phi_{q}, \\
& \Psi_{b}=\sqrt{1-m^{2}} \Phi_{D}-m \Phi_{q}, \quad 0<m<1,
\end{aligned}
$$

where $\Phi_{q}$ and $\Phi_{D}$ are the wave functions of the KS single and double excitations, respectively. The fraction $m$ determines how much double excitation character the true excitations have: for $m^{2}>1 / 2$, we may say that $\Psi_{a(b)}$ is predominantly double(single), and vice-versa. We wish to construct the exact xc kernel at frequencies near this multiplet. For frequencies near $\omega_{q}$, the Lehman sum in $\chi_{s}$ is dominated by $\Phi_{q}$ :

$$
\chi_{s}\left(\mathbf{r}, \mathbf{r}^{\prime} ; \omega\right) \approx \frac{A\left(\mathbf{r}, \mathbf{r}^{\prime} ; \omega\right)}{\omega-\omega_{q}},
$$

where the matrix $A\left(\mathbf{r}, \mathbf{r}^{\prime} ; \omega\right)$ is only very weakly frequency dependent: $\quad A\left(\mathbf{r}, \mathbf{r}^{\prime} ; \omega\right)=\phi_{i}^{*}(\mathbf{r}) \phi_{a}(\mathbf{r}) \phi_{i}\left(\mathbf{r}^{\prime}\right) \phi_{a}^{*}\left(\mathbf{r}^{\prime}\right)+O(\omega$ $\left.-\omega_{q}\right)$. For the interacting system at $\omega$ near $\omega_{q}($ and $\lambda \rightarrow 0)$,

$$
\chi\left(\mathbf{r}, \mathbf{r}^{\prime} ; \omega\right) \approx A\left(\mathbf{r}, \mathbf{r}^{\prime} ; \omega\right)\left(\frac{1-m^{2}}{\omega-\omega_{a}}+\frac{m^{2}}{\omega-\omega_{b}}\right) \text {. }
$$

Both interacting states in the multiplet contribute to $\chi$, whereas only the single excitation of the KS multiplet contributes to $\chi_{s}$, as discussed earlier. Note that Eqs. (7) and (8) give the behavior of the exact susceptibilities at frequencies near $\omega_{q}$. The oscillator strength of the KS single excitation is shared between the two interacting levels, in a ratio determined by how much double excitation is mixed in [see Eq. (8)], with the predominantly single state being brighter. We now define a dressed SPA (DSPA) as

$$
\omega=\omega_{q}+2\left[q\left|f_{\mathrm{HXC}}(\omega)\right| q\right],
$$

where here frequency dependence is retained in the xc kernel [cf. Eq. (5)]. Using Eq. (1) in the DSPA Eq. (9), we write

$$
\omega=\omega_{q}+2\left(\left[q\left|\chi_{s}^{-1}\right| q\right]-\left[q\left|\chi^{-1}\right| q\right]\right) .
$$

Substituting now Eqs. (7) and (8), and requiring that the solutions $\omega$ equal the true interacting frequencies $\omega_{a}$ and $\omega_{b}$, determines that the matrix element $[q|A| q]$ $\equiv \int d \mathbf{r} d \mathbf{r}^{\prime} A\left(\mathbf{r}, \mathbf{r}^{\prime}, \omega\right) \phi_{i}^{*}(\mathbf{r}) \phi_{a}(\mathbf{r}) \phi_{i}\left(\mathbf{r}^{\prime}\right) \phi_{a}^{*}\left(\mathbf{r}^{\prime}\right)=2$. Equation (10) then tells us that for frequencies near $\omega_{q}, f_{\mathrm{HXC}}$ has frequency dependence given by

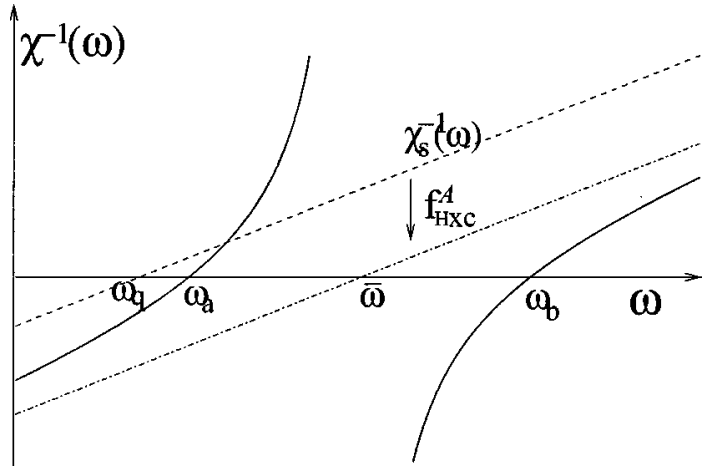

FIG. 1. Frequency dependence near a double excitation (see the text): Near a single excitation, $\chi_{s}^{-1}(\omega)$ (upper dashed line) has one zero at the KS transition $\omega_{q}$, which an adiabatic kernel $f_{\mathrm{HXC}}^{A}$ shifts to $\bar{\omega}$ [Eq. (12)]. Frequency dependence of Eq. (11) gives the exact $\chi^{-1}(\omega)$ (solid line) two zeroes at the transition frequencies $\omega_{a}, \omega_{b}$ of the true mixed single and double states.

$$
2\left[q\left|f_{\mathrm{HXC}}(\omega)\right| q\right]=\left(\bar{\omega}-\omega_{q}\right)+\frac{\bar{\omega}^{\prime} \bar{\omega}-\omega_{a} \omega_{b}}{\left(\omega-\bar{\omega}^{\prime}\right)},
$$

where $\bar{\omega}\left(\bar{\omega}^{\prime}\right)$ averages the true excitations weighted toward the predominantly single(double) excitation:

$$
\begin{aligned}
& \bar{\omega}^{\prime}=m^{2} \omega_{a}+\left(1-m^{2}\right) \omega_{b}, \\
& \bar{\omega}=\left(1-m^{2}\right) \omega_{a}+m^{2} \omega_{b} .
\end{aligned}
$$

Equation (11) is exact: it shows the behavior of the exact xc kernel as a function of frequency, at frequencies near $\omega_{q}$, when a double excitation interacts with an otherwise isolated single. This is illustrated in Fig. 1. The first term on the right-hand side of Eq. (11) is frequency independent and describes exchange and a frequency-averaged correlation contribution; it is of the order of the interaction strength $\lambda$ (and higher). The second term contains the rest of the correlation effects, and is of order $\lambda^{2}$ and higher: this is strongly frequency dependent.

ATDDFT approximates only the first term in Eq. (11): at best, it yields the weighted average toward the state of predominantly single excitation character, $\bar{\omega}$. For states that include only a small fraction of double excitations, ATDDFT performs well but as the double excitation component increases, it begins to deviate. For example, if the two levels are 50:50 mixtures of single and double, then ATDDFT gives one energy approximately halfway between the true energies. If the true energies are close then ATDDFT will appear to give a good estimate for the state which has significant double excitation character, just because it is close to the single-dominated state. However, when there is strong mixing and the levels are not close to each other, then ATDDFT does not give accurate results for either excitation. Use of this model provides a better understanding of the results in Refs. 23 and 25.

The frequency dependence of Eq. (11) is a central result of this paper: substituting it in the DSPA Eq. (9) recovers (by construction) the exact interacting transition frequencies of states that are mixtures of a single and a double. Equation (11) shows the frequency-dependent behavior that the exact $\mathrm{xc}$ kernel must have when a double excitation is close to a 
single excitation, well separated from all others. We next derive the $\mathrm{xc}$ kernel for this situation, to be used in the vicinity of these states, that uses only input from an adiabatic TDDFT calculation and becomes exact in the weakinteraction limit.

\section{THE FREQUENCY-DEPENDENT KERNEL: WEAK INTERACTION LIMIT AND BEYOND}

Consider a situation where one has a KS singly excited state $\Phi_{q}$ and a doubly excited state $\Phi_{D}$ much closer in energy to each other than to all the other states. In terms of the KS single-particle orbitals, $\Phi_{q}$ is a Slater determinant replacing one orbital $\phi_{i}$ occupied in the ground state with an unoccupied one $\phi_{a} . \Phi_{D}$ replaces two orbitals occupied in the ground state $\phi_{j}, \phi_{k}$ with two unoccupied, $\phi_{b}, \phi_{c}$. The orbital energies are such that $\epsilon_{b}+\epsilon_{c}-\epsilon_{j}-\epsilon_{k} \approx \epsilon_{a}-\epsilon_{i}$.

We shall now derive, in three steps, a nonempirical approximation for the frequency-dependent xc kernel that captures both the interacting states which are mixtures of the KS double and single states. Our result is exact in the weakinteraction limit.

(i) Diagonalizing the Hamiltonian in this $2 \times 2$ block, we find

$$
E=H_{q q}+\frac{\left|H_{q D}\right|^{2}}{E-H_{D D}},
$$

where $H_{q q}=\left\langle\Phi_{q}|H| \Phi_{q}\right\rangle$ is the expectation value of the Hamiltonian $H=T+V_{\text {ee }}+V_{\text {ext }}$ taken in the KS singly excited state $\Phi_{q}$. Here, $T$ is the kinetic energy operator, $V_{\text {ee }}$ is the two-body electron-electron interaction $\left(1 /\left|\mathbf{r}_{1}-\mathbf{r}_{2}\right|\right)$, and $V_{\text {ext }}$ is the external potential on the electrons due to the nuclear attraction. Similarly $H_{D D}$ is the expectation value taken in the doubly excited state. $H_{q D}$ is the matrix element $\left\langle\Phi_{q}|H| \Phi_{D}\right\rangle$. (Note that if a double excitation mixes strongly with several singles, ${ }^{32}$ one would fold into Eq. (13) the row and column of the double into the basis spanned by the singles: this amounts to a "partitioning" technique known in configuration-interaction methods ${ }^{34}$.)

This gives the exact energies when the single and double pair are truly isolated from all the other excitations in the system. It also gives the exact energies when the pair is well separated (albeit not infinitely so) from the other excitations in the limit of weak electron-electron interaction strength $\lambda \rightarrow 0$ : in this limit, the matrix elements are those of the $T$ $+\lambda\left(V_{\mathrm{ee}}-v_{\mathrm{H}}-v_{\mathrm{X}}\right)$, where $v_{\mathrm{H}}$ is the Hartree potential, and $v_{\mathrm{X}}$ is the exchange potential. In that case, Eq. (13) agrees with Görling-Levy perturbation theory ${ }^{35-37}$ when applied to near-degenerate states. ${ }^{38}$

(ii) Substituting $E=\omega+H_{00}$ in Eq. (13), where $H_{00}$ is the expectation value of the Hamiltonian in the KS ground state, then yields an expression for the transition frequency. For consistency, $H_{00}$ is subtracted rather than the true ground-state energy so as not to unbalance the errors arising from correlation resulting from the original truncation of $H$ in the $2 \times 2$ basis. This would give

$$
\omega=\left(H_{q q}-H_{00}\right)+\frac{\left|H_{q D}\right|^{2}}{\omega-\left(H_{D D}-H_{00}\right)} .
$$

(iii) Finally we now replace the frequency-independent term by the SPA, using an adiabatic kernel $f_{\mathrm{XC}}^{A}$,

$$
H_{q q}-H_{00} \rightarrow \omega_{q}+2\left[q\left|f_{\mathrm{H}}+f_{\mathrm{XC}}^{A}\right| q\right],
$$

where $\omega_{q}=\epsilon_{a}-\epsilon_{i}$ and $[q|f| q]$ is defined as in Eq. (4). In this way we recover the exchange-correlation of ATDDFT in the limit of weak coupling to the double excitation. We thus obtain an expression for the xc kernel for frequencies near $\omega_{q}$, of the form of the DSPA Eq. (9), with

$$
2\left[q\left|f_{\mathrm{XC}}(\omega)\right| q\right]=2\left[q\left|f_{\mathrm{XC}}^{A}\left(\omega_{q}\right)\right| q\right]+\frac{\left|H_{q D}\right|^{2}}{\omega-\left(H_{D D}-H_{00}\right)} .
$$

This is the second main result of this paper. It is exact for well-separated poles in the weak-interaction limit and there are no empirical parameters. The kernel of Eq. (15) is to be used as an a posteriori correction to ATDDFT: one would first perform a TDDFT calculation using an adiabatic kernel, then scan the KS single excitations to see if the sum of any two lies close to any of the singles. If so, one would recompute the transition frequencies only of this particular pair using Eq. (15) in Eq. (9). In another paper, we will show how this idea may be generalized to the case where more than one single excitation interacts with the double excitation; ${ }^{32}$ we are thus able to capture the significantly doubly excited $2^{1} A_{g}$ states of butadiene and hexatriene.

\section{DEMONSTRATION ON A SIMPLE MODEL}

Here, we demonstrate our results on a simple, exactly solvable model system: two one-dimensional fermions in a parabolic external potential, interacting via a delta-function repulsion of strength $\lambda$ :

$$
H=-\frac{1}{2}\left(\frac{d^{2}}{d x_{1}^{2}}+\frac{d^{2}}{d x_{2}^{2}}\right)+\frac{1}{2} k\left(x_{1}^{2}+x_{2}^{2}\right)+\lambda \delta\left(x_{1}-x_{2}\right) .
$$

Transforming to center of mass and relative coordinates $R$ $=\left(x_{1}+x_{2}\right) / 2, u=x_{1}-x_{2}$, the Schrödinger equation decouples into two separate equations: that in $R$ is a simple harmonic oscillator, and that in $u$ can be solved numerically. The exact eigenstates are characterized by the quantum numbers of excitation in the $R$ and $u$ coordinates, $\{J j\}$ respectively. Note that the energy of any pure $R$ excitation $\{J 0\}$ is given by the harmonic oscillator expression $\sqrt{k}(J+1 / 2)$; that for a pure $u$ excitation $\{0 j\}$ is larger than $\sqrt{k}(j+1 / 2)$ due to the inter-particle repulsion. We consider here only singlet states, which means $j$ is even (since with $j$ odd, the spatial state is antisymmetric). The multiplet structure of the levels is found by grouping together the singlet states that are degenerate when the interaction is turned off $(\lambda=0)$. In the first column of Fig. 2, we plot the transition frequencies to the lowest five excited states, $(\{10\},\{02\},\{20\},\{12\},\{30\})$, for interaction strength $\lambda=0.2$.

From the exact ground-state density, we find the exact KS potential by inverting the KS equation for the two electrons; in the ground state they both occupy the lowest spatial orbital. We solve for all the excited KS orbitals numerically; the frequencies to these levels are shown in the second col- 


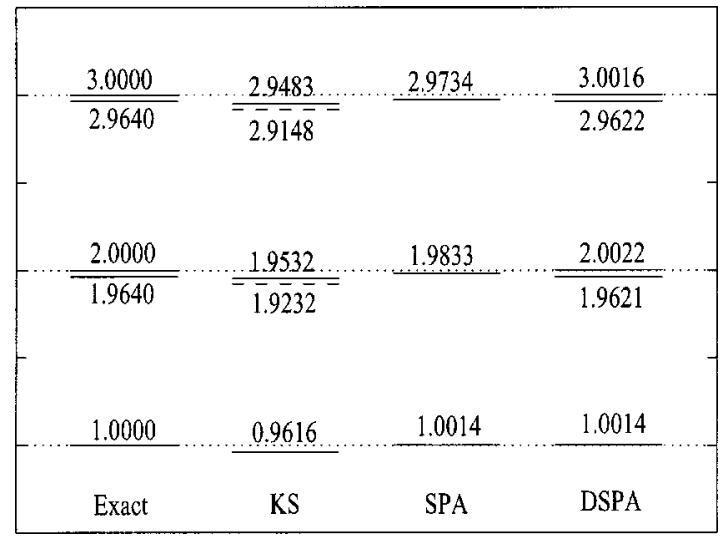

FIG. 2. Transition frequencies (in hartrees) of the lowest three excited multiplets of our test system with $k=1$ (see the text), at interaction strength $\lambda=0.2$. First column: exact transition frequencies of the states $\{1,0\},\{0,2\}$, $\{2,0\},\{1,2\},\{3,0\}$, in ascending order. Second column: KS levels with single excitations solid, and doubly excited states dashed. Third column: SPA using exact-exchange corrects the KS singles toward the weighted average of the true multiplet (see the text), and loses the distinction between the two states in each multiplet. Fourth column: our frequency-dependent DSPA approximation, Eq. (15), recovers all states with good accuracy.

umn in Fig. 2. The solid lines correspond to single excitations where, in ascending order, the first, second and third excited KS orbital are singly occupied. The dashed lines are double excitations: the lower dashed line is when both the fermions occupy the first excited orbital and the higher dashed line is when both the first and second excited orbitals are occupied. The dashed lines do not appear in the KS linear response as discussed earlier. The true levels in the second and third excited multiplets are thus mixed single and double excitations.

The third column contains the results from an adiabatic SPA calculation. Because the interaction strength $\lambda$ is small, we approximate $f_{\mathrm{XC}}$ by exact exchange, neglecting any correlation $\left(f_{\mathrm{X}}\right.$ is of the order of $\lambda$, but $f_{\mathrm{C}}$ is of the order of $\left.\lambda^{2}\right)$. For two electrons,

$$
f_{\mathrm{HXC}}^{A} \approx f_{\mathrm{HX}}=\frac{1}{2} f_{\mathrm{H}}=\frac{\lambda}{2} \delta\left(x_{1}-x_{2}\right) .
$$

The SPA using this kernel is almost exact for pure singles, because the interaction is weak and KS multiplets are well separated, as demonstrated by its accuracy for the first excited state.

The SPA fails for the second and third excited multiplets due to the mixing of the KS single and double in each case, producing only one level in each multiplet. The interaction couples each pair of KS states in the multiplet. Consider the second excited multiplet, containing two KS states: a single excitation into the second excited KS orbital, and a double excitation into the first excited $\mathrm{KS}$ orbital. The interaction transforms each into a 50:50 mixture of a single and a double, in the $\lambda \rightarrow 0$ limit. Only one level, which approximates the weighted average $\bar{\omega}$ [Eq. (12)] can be obtained using ATDDFT, as shown here in the SPA column: this level lies almost in the middle of the two true levels $\left(m^{2}=0.5\right)$. Our DSPA of Eq. (15) in the fourth column recaptures both levels, approximating the true levels closely. The third mul- tiplet contains a KS single excitation into the third excited KS orbital, and a double excitation into the first and second KS orbitals. A weak interaction couples the single excitation with the double, producing one state, $\{30\}$, of $\frac{1}{3}: \frac{2}{3}$ mixture of single to double, and the other state, $\{12\}$, of $\frac{2}{3}: \frac{1}{3}$ mixture. The SPA yields one state of frequency approximating $\bar{\omega}$ with $m$ $=1 / \sqrt{3}$. Again, our DSPA recovers both of the true levels very accurately.

In this example, we chose $\lambda \ll 1$ to ensure that the system is weakly correlated, and that perturbation theory is accurate. The important point of our results is that DSPA provides as accurate results for the mixed states as ATDDFT does for single excitations. In fact, as $\lambda \rightarrow 0$, both become exact in their own domains, but only DSPA can accommodate double excitations. Thus DSPA is the correct generalization of ATDDFT to include mixtures of single and double excitations.

\section{DISCUSSION AND CONCLUDING REMARKS}

In recent work $^{24}$ transitions to states of significant double-excitation character in linear polyene oligomers were found to be better approximated using ATDDFT than in a configuration-interaction singles calculation. It was also recognized there that the calculations underestimated the lowest singlet single excitation and results were basis dependent. Our results show the severe frequency-dependence in the xc kernel that is needed to yield a consistent treatment of excitations in this system. We have tested our approximation on the lowest excitations of butadiene and hexatriene, ${ }^{32}$ producing much improved (and consistent) transition frequencies for the lowest excitations.

In summary, in order to describe states of double excitation character accurately, frequency dependence is essential in the exchange-correlation kernel of TDDFT. We have shown exactly what this frequency dependence is in the vicinity of a double excitation mixing with a single excitation and derived, and successfully tested, an approximation for it. It is proposed that future calculations could proceed as follows: (i) solve the linear response equations using an adiabatic kernel, (ii) determine whether double excitations mix in by, for example, scanning the KS single excitations to see if the sum of any two of them lies close to any of them, and (iii) then apply our DSPA correction [Eq. (15) in Eq. (9)] just to the single that is coupled to the double. This should provide accurate approximations to states of double excitation character, when a KS double mixes closely with one single, and is well separated from the other levels (cf. conditions in Ref. 15 for the SPA), while leaving the treatment of largely single excitation states unchanged from ATDDFT. Although presented here in a single-pole framework, our kernel can be generalized to the case when several single excitations lie close to and mix with a double. ${ }^{32}$

\section{ACKNOWLEDGMENTS}

We thank the National Science Foundation (Grant No. CHE-9731634), the Petroleum Research Fund, and the Office of Naval Research (Grant No. NOOOO14-01-1-1061) 
for support. R.J.C. gratefully acknowledges support from the Rutgers University Nanochemistry Consortium. We thank Todd Martinez for useful discussions.

${ }^{1}$ W. Kohn, Rev. Mod. Phys. 71, 1253 (1999).

${ }^{2}$ P. Hohenberg and W. Kohn, Phys. Rev. 136, B864 (1964).

${ }^{3}$ W. Kohn and L. J. Sham, Phys. Rev. 140, A1133 (1965).

${ }^{4}$ J. P. Perdew, K. Burke, and M. Ernzerhof, Phys. Rev. Lett. 77, 3865 (1996); 78, 1396 (1997(E)).

${ }^{5}$ A. Becke, J. Chem. Phys. 98, 5648 (1993).

${ }^{6}$ J. P. Perdew, S. Kurth, A. Zupan, and P. Blaha, Phys. Rev. Lett. 82, 2544 (1999); 82, 5179 (1999(E)).

${ }^{7}$ J. L. F. Da Silva, C. Stampfl, and M. Scheffler, Phys. Rev. Lett. 90, 066104 (2003).

${ }^{8}$ E. Runge and E. K. U. Gross, Phys. Rev. Lett. 52, 997 (1984).

${ }^{9}$ M. Petersilka, U. J. Gossmann, and E. K. U. Gross, Phys. Rev. Lett. 76, 1212 (1996).

${ }^{10}$ M. E. Casida, in Recent Developments and Applications in Density Functional Theory, edited by J. M. Seminario (Elsevier, Amsterdam, 1996).

${ }^{11}$ K. Yabana and G. F. Bertsch, Phys. Rev. B 54, 4484 (1996).

${ }^{12}$ K. Yabana and G. F. Bertsch, Int. J. Quantum Chem. 75, 55 (1999).

${ }^{13}$ A. Tsolakidis, D. Sánchez-Portal, and R. M. Martin, Phys. Rev. B 66, 235416 (2002).

${ }^{14}$ N. T. Maitra, K. Burke, H. Appel, E. K. U. Gross, and R. van Leeuwen, in Reviews in Modern Quantum Chemistry: A Celebration of the Contributions of R. G. Parr, edited by K. D. Sen (World-Scientific, Singapore, 2002).

${ }^{15}$ H. Appel, E. K. U. Gross, and K. Burke, Phys. Rev. Lett. 90, 043005 (2003).

${ }^{16}$ M. E. Casida, C. Jamorski, K. C. Casida, and D. R. Salahub, J. Chem. Phys. 108, 4439 (1998).

${ }^{17}$ D. J. Tozer and N. C. Handy, J. Chem. Phys. 109, 10180 (1998).
${ }^{18}$ G. Vignale, Phys. Rev. Lett. 74, 3233 (1995).

${ }^{19}$ G. Vignale and W. Kohn, Phys. Rev. Lett. 77, 2037 (1996).

${ }^{20}$ N. T. Maitra, K. Burke, and C. Woodward, Phys. Rev. Lett. 89, 023002 (2002).

${ }^{21}$ I. V. Tokatly and O. Pankratov, Phys. Rev. B 67, 201103(R) (2003).

${ }^{22}$ D. J. Tozer and N. C. Handy, Phys. Chem. Chem. Phys. 2, 2117 (2000).

${ }^{23}$ D. J. Tozer, R. D. Amos, N. C. Handy, B. O. Roos, and L. Serrano-Andres, Mol. Phys. 97, 859 (1999).

${ }^{24}$ C. Hsu, S. Hirata, and M. Head-Gordon, J. Phys. Chem. A 105, 451 (2001).

${ }^{25}$ S. Hirata and M. Head-Gordon, Chem. Phys. Lett. 302, 375 (1999).

${ }^{26}$ E. K. U. Gross, J. F. Dobson, and M. Petersilka, Top. Curr. Chem. 181, 81 (1996).

${ }^{27}$ See, e.g., R. H. Cave, in Modern Electronic Structure Theory and Applications in Organic Chemistry, edited by E. R. Davidson (World, Singapore, Singapore, 1997), p. 197.

${ }^{28}$ J. Lappe and R. J. Cave, J. Phys. Chem. A 104, 2294 (2000).

${ }^{29}$ J. D. Watts, S. R. Gwaltney, and R. J. Bartlett, J. Chem. Phys. 105, 6979 (1996).

${ }^{30}$ L. Serrano-Andres, M. Merchan, I. Nebot-Gil, R. Lindh, and B. O. Roos, J. Chem. Phys. 98, 3151 (1993).

${ }^{31}$ B. Hudson and B. Kohler, Annu. Rev. Phys. Chem. 25, 437 (1974).

${ }^{32}$ R. J. Cave, F. Zhang, N. T. Maitra, and K. Burke, Chem. Phys. Lett. (to be published).

${ }^{33}$ T. Grabo, M. Petersilka, and E. K. U. Gross, THEOCHEM 501, 353 (2000).

${ }^{34}$ I. N. Levine, Quantum Chemistry, 4th ed. (Prentice-Hall, Englewood Cliffs, NJ, 1991).

${ }^{35}$ A. Görling and M. Levy, Phys. Rev. B 47, 13105 (1993).

${ }^{36}$ X. Gonze and M. Scheffler, Phys. Rev. Lett. 82, 4416 (1999).

${ }^{37}$ C. Filippi, C. J. Umrigar, and X. Gonze, J. Chem. Phys. 107, 9994 (1997).

${ }^{38}$ F. Zhang and K. Burke, Phys. Rev. A (to be published). 